\title{
A Multiple Fiber Grating Sensor System using Code Division Multiple Access
}

\author{
Li Wei $^{1, a}$,Zhang Yongjia ${ }^{1, b}$, Wen Hongqiao ${ }^{1}$ \\ ${ }^{1}$ Fiber Optic Sensing Technology Research Center , Wuhan University Of Technology, \\ No.122,Luoshi Road, Hongshan District, Wuhan430070, China \\ alimighty@yahoo.com.cn, ${ }^{\mathrm{b}} 4421977 @ q q . c o m$
}

Key words: FBG; CDMA; PRBS

\begin{abstract}
In order to increase the multiplying density of Fiber Bragg Grating (FBG) sensors, a novel FBG Sensing System based on CDMA technology has been developed. Simulation experiment indicates the CDMA technology combine with optical fiber grating sensing system together successfully. Furthermore, the system can distinguishes the FBG and enhance the FBG network band utilization.
\end{abstract}

\section{Introduction}

Fiber Bragg gratings(FBG), which are widely used in optical communications, have also been studied actively for sensor applications, including the measurement of strain, pressure, temperature, and vibrations. FBG sensors have the common advantages of optical fiber based devices such as low propagation loss, light weight, durability against corrosion, and EMI immunity [1-3].

Among the different multiplexing techniques of FBG sensors, the wavelength-division multiplexing (WDM) technique is one of the most widely used [4-8]. The maximum number of multiplexed sensors are limited by the width of the broadband (or wavelength-scanned) light source and the Bragg wavelength operation ranges required by the FBG sensors. Another technique is the time-division multiplexing (TDM) technique [9,10], where a pulsed source is used to illuminate the gratings placed at different distances from the source. The reflected signals from the gratings are separated in time domain. These pulses arrive at a time determined by the distance between interrogator and FBG sensor, and one FBG sensor can be distinguished from another by analyzing the pulse arrival times. The pulse has to travel to the last FBG sensor in the sensor array and reflect back before another pulse can be sent from the source. This imposes a limit on the sensor detection speed, number of sensors, and maximum distance between sensors.

Further to these problem, we have brought up a successive scheme which use code division multiple access(CDMA) in this articles. The system utilizes the autocorrelation property to separate individual sensors from the multiplexed output of many sensors. To identify each sensor, a pseudo-random binary sequence (PRBS) code is sent as a random signal into the FBG sensor array, and the information of the corresponding sensor is decoded in the electrical domain. Simulation experiment indicates the CDMA technology combine with optical fiber grating sensing system together successfully. Furthermore, the system can distinguishes the FBG and enhance the FBG network band utilization.

\section{Principle of operation}

A proposed conceptual diagram of a CDMA sensor network is shown in Figure 1. To cover the whole wavelength band of all sensors, a broadband light source is used. The broadband source was directly modulated by using PRBS codes. The modulated optical signal from broadband light source is amplified by using erbium doped fiber amplifier(EDFA) and goes through FBG sensors via circulator. The FBG sensors were used for measuring strain sensitivity. 


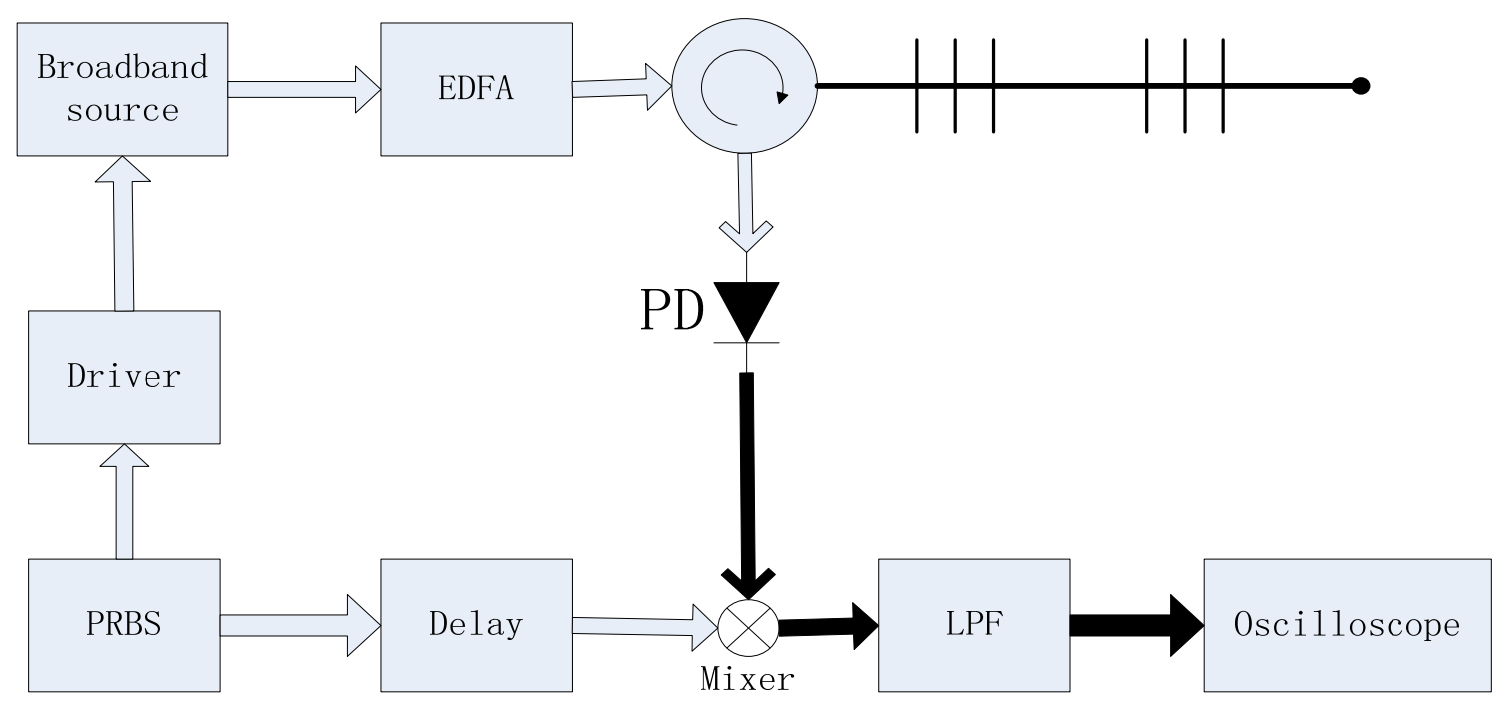

Fig.1 Fiber Bragg Grating sensing System Using CDMA

When the modulated optical signal experienced FBG sensor array, the optical signal which was consistent with center wavelength of FBG sensors is reflected and added from each sensors. The optical signal is converted into electrical signal by using photodetector (PD). Then, the signal was correlated with delayed PRBS code that has the same data pattern. At the end, the result was monitored by an oscilloscope. There are two peak values in the output traces of the integrator, which correspond to the signals reflected from each sensor and vary according to the applied strains. Variations of peak values were measured by use of an oscilloscope.

In the CDMA sensor networks, the autocorrelation function, $\psi(\tau)$ with a reflected signal from a sensor is given by

$$
\psi(\tau)=\int_{-\infty}^{+\infty} f(t-\tau) \cdot f^{\prime}\left(t-\tau_{s}\right) d t
$$

Where $f(t)$ is the modulation signal, $f^{\prime}(t)$ is the received signal, $\tau$ is time shift by electric delay of reference signal and $\tau_{s}$ is propagation delay from a sensor to detection circuit. The autocorrelation function can be obtained when the time shift of reference $\tau$ has the same value as the time delay of a sensor $\tau_{s}$. By measuring this value, each sensor is monitored.

The PRBS generator makes a sequence by using an m-stage linear feedback shift register generator .The sequence length is $2^{m}-1$. It indicates that the number of FBG sensors is $2^{m}-1$. Every sensor has its own PRBS.

\section{Experiment results}

In this experiment, we assume that the two kinds of circumstances. The first is that two FBG sensors which have the different center wavelength and different reflectivity . Two FBG sensors were used for a simple demonstration of applied strain. The center wavelengths of the sensors were 1,551.8, 1,552.6 nm, respectively. The bandwidth of each sensor was $0.2 \mathrm{~nm}$ and the reflectivity of each sensor was 90\%, 50\%. Figure 2 shows the spectral responses of Bragg grating sensor1 and sensor2. Figure 3 shows changes of the center wavelength according to pressure. When a strain is applied to the sensors, the reflected from the sensors signals are detected by a photodiode. In the figure 3 , the center wavelength of sensor 1 changed because we applied strain to sensor 1 . 


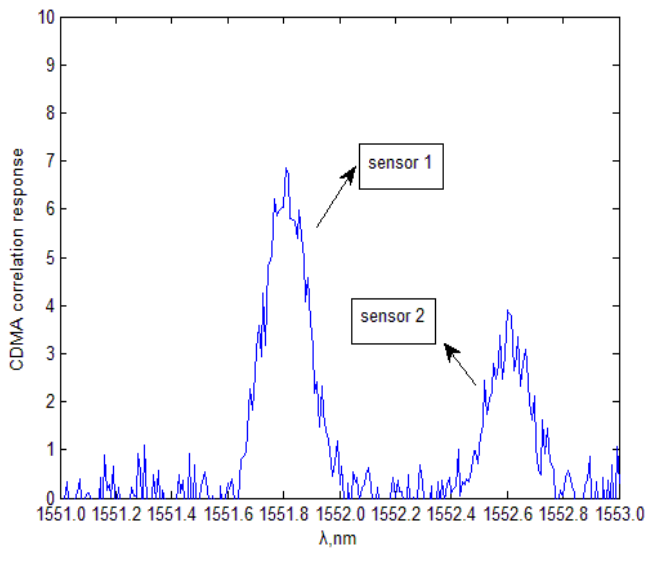

Fig.2 Spectral responses of Bragg and sensor2,and for sensor1 with strain

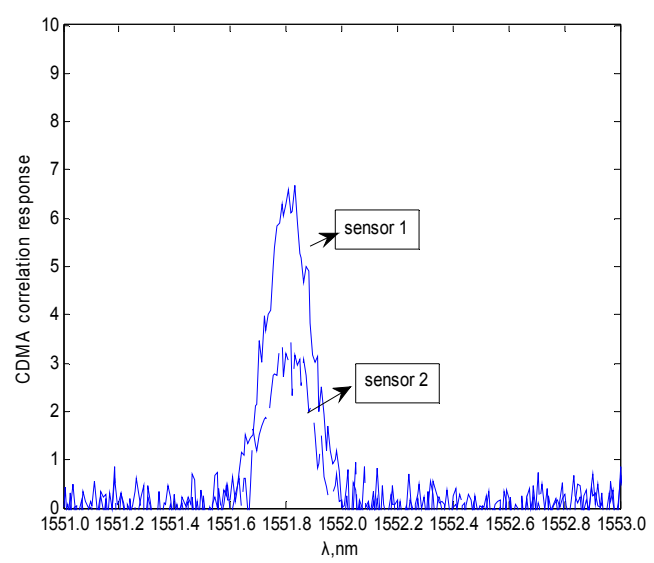

Fig.4 the theoretical model of Spectral and sensor2 by OSC

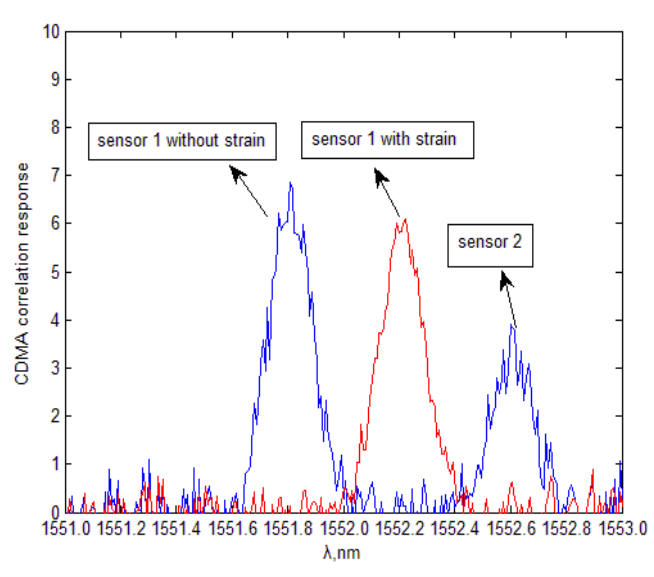

Fig.3 Spectral responses of Bragg grating sensor1 grating sensor1 and sensor2

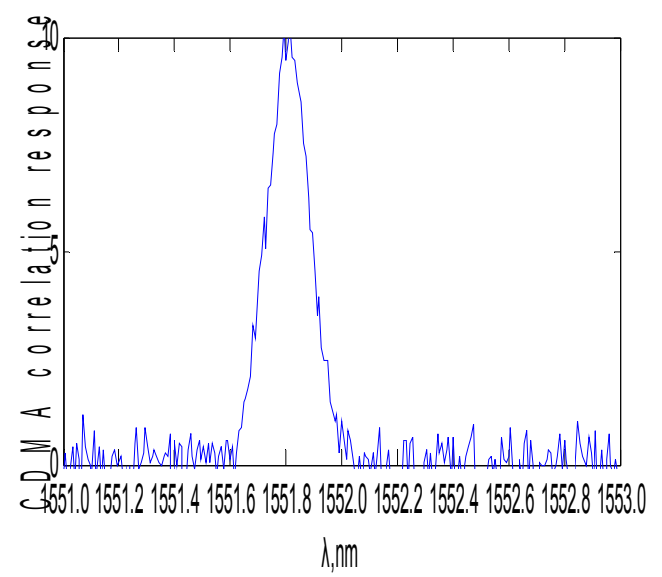

Fig.5 Spectral responses of Bragg grating sensor1 responses for Bragg grating sensor1 and sensor2

Another kind of circumstance is that the two FBG sensors have the same center wavelength. The theoretical model of spectral responses is figure 4, because they have the same center wavelength. The spectral responses of two FBG overlap in practice. Figure 5 shows spectral responses of Bragg grating sensor1 and sensor2 by OSC without CDMA. We could not separate which spectral responses of Bragg grating is sensor1 or sensor2. But in our experiment, autocorrelation method was used to separate each sensor. Figure 6 and Figure 7 show the spectral responses of separated Bragg grating sensor1 and sensor2 with CDMA.
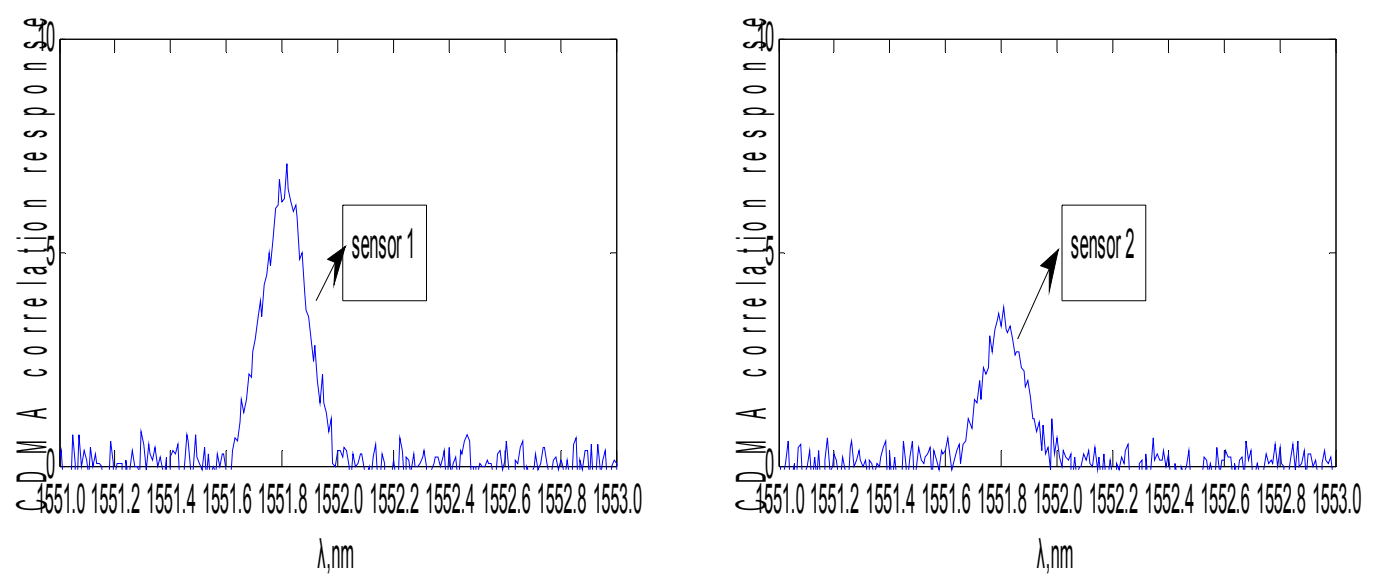

Fig.6 Spectral responses of Bragg grating sensor1 Fig.7 Spectral responses of Bragg grating sensor2 


\section{Conclusion}

The FBG spectra can overlap and do not need to be highly accurate. A pulse train representing a unique code is send into the network and will be reflected with a time shift at each sensor. Two pulses can directly follow after each other, what solves the information deficit of TDMA. Modulating the signal at the receiver side by the correct time adjusted code and integrating it over time, one gets back the sensor information plus some smaller distortions from other sensors.

An FBG sensor network based on CDMA and its simulation was shown. In this article, First we use two FBG with different center wavelength and reflectivity for a simple demonstration of applied strain. The simulation proves the correctness of the theory based on CDMA. Second we use two FBG with same center wavelength and different reflectivity. In this experiment, the autocorrelation method of CDMA was used to separate each sensor.

\section{Acknowledgments}

This work is supported by the National Natural Science Foundation of China (No.61205070).

\section{References}

[1]. A. D. Kersey, Davis, M.A. ， Patrick, H.J. ， LeBlanc, M. ， Koo, K.P. ， Askins, C.G. ， Putnam, M.A. , Friebele, E.J. , “Fiber grating sensors,” Lightwave Technology.15, 1442(1997). [2]. G. D. Lloyd, L. A. Everall, K. Sugden, I. Bennion "Resonant cavity time-division-multiplexed fiber Bragg grating sensor interrogator, ”Photonics Technology Letters.16, 232(2004).

[3]. M.G.Xu, H. Geiger, and J. P. Dakin. "Modeling and performance analysis of a fiber Bragg grating interrogation system using an acousto-optic tunable filter," Lightwave Technology.14, 391(1996).

[4]. Yun Jiang Rao, D. J . Web b, D. A. Jack son, "In fiber Bragg grating temperature sensor system for medical applications,” Lightwave Technology.15, 779(1997).

[5]. Gong, J.M. MacAlpine, J.M.K.,“A novel wavelength detection technique for fiber Bragg grating sensors,”. Photonics Technology Letters.14,678(2002).

[6]. R. D. Feldman, "Crosstalk and loss in wavelength division multiplexed systems employing spectral slicing,” Lightwave Technology.15, 1823(1997).

[7]. R. D. Feldman, E. E. Harstead, S. Jiang, “An evaluation of architectures incorporating wavelength division multiplexing for broad-band fiber access,” Lightwave Technology.16, , 16: 1546(1998).

[8]. J. B. Stark, M. C. Nuss, W. H. Knox ,“Cascaded WDM passive optical network with a highly shared source,” Photonics Technology Letters.9, 1170(1997).

[9]. David J.F. Cooper et al., "Time-division multiplexing of large serial fiber-optic Bragg grating sensor arrays,”, Applied Optics.40, 2643(2001).

[10]. David J.F. Cooper et al., "Simple and Highly Sensitive Method for Wavelength Measurement of Low-Power Time-Multiplexed Signals Using Optical Amplifiers”, J-LT.21, 1612(2003). 